\title{
Temperature effects in the collisional deactivation of highly vibrationally excited pyrazine by unexcited pyrazine
}

\author{
Laurie A. Miller \\ Department of Chemistry, University of Michigan, Ann Arbor, Michigan 48109-2143 \\ Carolyn D. Cook \\ Department of Chemical Engineering, University of Michigan, Ann Arbor, Michigan 48109-2143 \\ John R. Barker ${ }^{\text {a) }}$ \\ Department of Chemistry and Department of Atmospheric, Oceanic, and Space Sciences, \\ University of Michigan, Ann Arbor, Michigan 48109-2143
}

(Received 25 April 1996; accepted 17 May 1996)

\begin{abstract}
Time-dependent infrared fluorescence (IRF) from the $\mathrm{C}-\mathrm{H}$ fundamental and overtone bands was used to monitor the vibrational deactivation (by unexcited pyrazine) of pyrazine excited at $308 \mathrm{~nm}$ with a pulsed laser. The 1-color and 2-color IRF results were modeled with collisional master equation calculations in order to determine the temperature dependence of the energy transfer parameters. The experimental data cannot be modeled without invoking a biexponential collision step size distribution, which implies that "super collisions" are significant. The results show that the energy transfer parameters are essentially constant at temperatures greater than the LennardJones well depth, but at lower temperatures, energy transfer is enhanced. It is likely that vibrationvibration energy transfer dominates in this system. (C) 1996 American Institute of Physics.
\end{abstract} [S0021-9606(96)02632-3]

\section{INTRODUCTION}

In a recent series of papers, new experimental data and new analyses of older data have been reported for the unimolecular decomposition of several free radicals, including $\mathrm{C}_{2} \mathrm{H}_{5},{ }^{1,2} t-\mathrm{C}_{4} \mathrm{H}_{9},{ }^{3}$ and sec- $\mathrm{C}_{4} \mathrm{H}_{9}{ }^{4}$. The reactions were studied as functions of temperature, pressure, and collider gas identity, and the results were analyzed using the best current thermochemistry. Numerical solutions of the Master Equation were used to fit the data and the resulting values of $\langle\Delta E\rangle_{d}$ (the average step size in deactivating collisions) are significantly temperature dependent. For example, $\langle\Delta E\rangle_{d}$ for the deactivation of vibrationally excited ethyl radicals by helium is proportional to $T^{n}$, where $1 \leqslant n \leqslant 1.6 .{ }^{1}$ This relatively strong temperature dependence is surprising, because measurements with the time-resolved infrared fluorescence (IRF) and the ultraviolet absorbance (UVA) methods found significantly weaker dependences for $\langle\Delta E\rangle_{d} \cdot{ }^{5-7}$ This difference may be due to the nature of the species investigated (free radicals vs stable aromatics and cycloheptatriene derivatives), to possible experimental errors, or to the analysis: the recent work on alkyl radicals did not consider the dependence of $\langle\Delta E\rangle_{d}$ on internal energy (although the effect should be small for small species). ${ }^{8}$

In the present work, the IRF method is used to investigate the vibrational deactivation of ground electronic state pyrazine as a function of temperature. The IRF method is a physical technique, as distinguished from unimolecular reaction studies. ${ }^{9-11}$ A number of benzene and toluene isotopomers have been investigated using single-color IRF and

a) Author to whom correspondence should be addressed; Electronic mail: jrbarker@umich.edu several of these species have been investigated using multicolor IRF, which provides more detailed information about the population distribution of excited molecules. ${ }^{12-19}$ In the present work, we used both one-color and two-color IRF, in which one or two IRF bands are monitored simultaneously. ${ }^{15,20}$

Pyrazine photophysics are very well known, ${ }^{21-24}$ because this species has been used extensively in studies of collision-free energy redistribution. ${ }^{25}$ Several groups have studied pyrazine collisional deactivation subsequent to photoexcitation, as discussed in a recent paper describing a onecolor IRF study of pyrazine deactivation by 19 collider gases at room temperature. ${ }^{26}$ In the present experiments, pyrazine is excited to the first singlet state via a $308 \mathrm{~nm}$ laser pulse and rapid intersystem crossing (ISC) produces ground electronic state pyrazine with $\sim 32500 \mathrm{~cm}^{-1}$ of vibrational energy. ${ }^{21,25}$ As the vibrationally excited molecules are collisionally deactivated, IRF from the $\mathrm{C}-\mathrm{H}$ stretch and its overtone are monitored. The IRF intensities were used in the present work to deduce the average vibrational energy of the excited population during the collisional cascade. ${ }^{7,12,27}$ Master equation simulations of the two IRF intensities were performed to obtain refined collision models and to deduce $\langle\Delta E\rangle_{d}$, the average energy transferred in each deactivating collision. Good simulations of the two-color IRF data could not be achieved, unless a biexponential step size distribution was employed.

\section{EXPERIMENT}

The IRF technique has been described in detail elsewhere. ${ }^{12,13,26}$ Gas phase pyrazine was irradiated by a $\mathrm{XeCl}$ excimer laser $(308 \mathrm{~nm})$ operating at a pulse repetition frequency of $25 \mathrm{~Hz}$. About $0.5 \%$ of the irradiated pyrazine 
was excited in each laser shot. ${ }^{26}$ Infrared emissions from the $\mathrm{C}-\mathrm{H}$ stretch near $3040 \mathrm{~cm}^{-1}$ and its overtone near 6000 $\mathrm{cm}^{-1}$ were monitored simultaneously through opposing sapphire side windows. The emission bands were spectrally isolated using broad-band interference filters. Signals from two InSb photovoltaic detectors (Infrared Associates) equipped with matched preamplifiers were amplified further with Tektronix AM-502 preamplifiers and recorded with the two channels of a LeCroy 9400 digital oscilloscope. The time response of the detection systems was $\sim 3 \mu \mathrm{s}$. Approximately 5000-10 000 laser shots were averaged for each run, and the data were transferred from the digital oscilloscope to a Macintosh computer for storage and analysis.

The cell was constructed from $20 \mathrm{~mm}$ i.d. $\times 31 \mathrm{~cm}$ long Pyrex tubing equipped with two windows on opposite sides. The cell was wrapped in several layers of copper foil and a helix of copper tubing was soldered to the foil; a second layer of foil covered the copper tubing in a "sandwich." Thermocouples were attached near each end of the cell and at the cell center above and below the two side windows. The copper jacket was wrapped with an electrical heating tape and the whole assembly was insulated with glass wool. The temperature was controlled above room temperature by flowing compressed air through the copper tubing and regulating the current in the heating tape with a proportional controller (Cole-Parmer) equipped with a platinum resistance thermometer. Below room temperature, cold gas from a liquid nitrogen source flowed at a constant rate through the copper tubing and the heating tape was used with the proportional controller, as before. The temperature could be held constant within $\pm 2{ }^{\circ} \mathrm{C}$ during a run and it was uniform over the central part of the cell within $\pm 5^{\circ} \mathrm{C}$.

Pyrazine (Sigma 99\%) was degassed in several freezepump-thaw cycles $(77 \mathrm{~K})$ before use. The experiments were performed using flowing conditions in order to minimize any potential accumulation of photodissociation products. A capacitance manometer (MKS Baratron, 0-10 Torr) was used to measure the pressure, which ranged from 20 mTorr to 90 mTorr. Below $\sim 20$ mTorr, diffusion from the field of view of the detector was observed to affect the IRF decay data. Pyrazine is known to photodissociate at short wavelengths, but the quantum yield for $\mathrm{HCN}$ production is only $\phi \approx 0.003$ under collision-free conditions ${ }^{24}$ and it will be smaller in a collisional system. ${ }^{28}$ Even if photodissociation products are present, they have very little residual vibrational energy and do not contribute significantly to the IRF.

\section{RESULTS AND DISCUSSION}

\section{Treatment of experimental data}

Examples of infrared fluorescence decay curves are presented in Fig. 1. The data from the first $\sim 3 \mu$ s were strongly affected by the detector rise time, so the IRF intensity (which includes a time-independent contribution from the cell walls) was extrapolated to $t=0$ using the expression:

$$
I_{i}=A_{i} \exp \left(-k_{i}^{\prime} t-b_{i}^{\prime} t^{2}\right)+G_{i}
$$

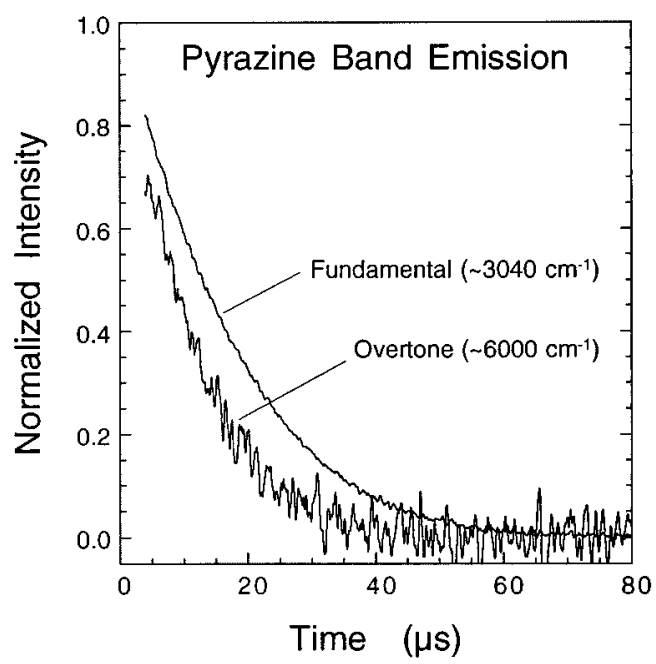

FIG. 1. IRF decays of the fundamental $\left(\sim 3040 \mathrm{~cm}^{-1}\right)$ and overtone $(\sim 6000$ $\mathrm{cm}^{-1}$ ) bands in neat pyrazine at $48 \mathrm{mTorr}$ and $273 \mathrm{~K}$.

where $k_{i}^{\prime}$ and $b_{i}^{\prime}$ are empirical parameters fitted by nonlinear least squares for the fundamental and overtone $(i=1,2)$. The data are normalized by setting $A_{i}=1$ and $G_{i}=0$. As the temperature is varied, the decay rates for both the fundamental and overtone bands increase, as illustrated in Fig. 2 for the fundamental band.

For convenience, the IRF decay curves were converted from a time scale to a collision scale according to the expression

$$
Z=\omega t=k_{\mathrm{LJ}} N_{p} t
$$

where $Z$ is the number of collisions, $k_{\mathrm{LJ}}$ is the LennardJones bimolecular collision rate constant,, 22 and $N_{p}$ is the number density of pyrazine. After converting to the collision scale, the IRF intensity can be described by a function similar to Eq. (1) with $t$ replaced by $Z$ and with new parameters $k_{i}$ and $b_{i}$ expressed in units of $Z^{-1}$ and $Z^{-2}$, respectively.

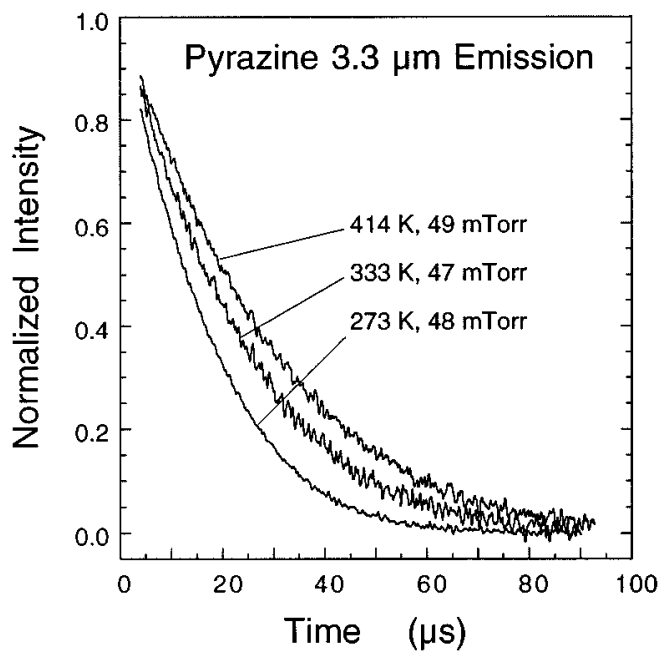

FIG. 2. IRF decays of the fundamental band. 


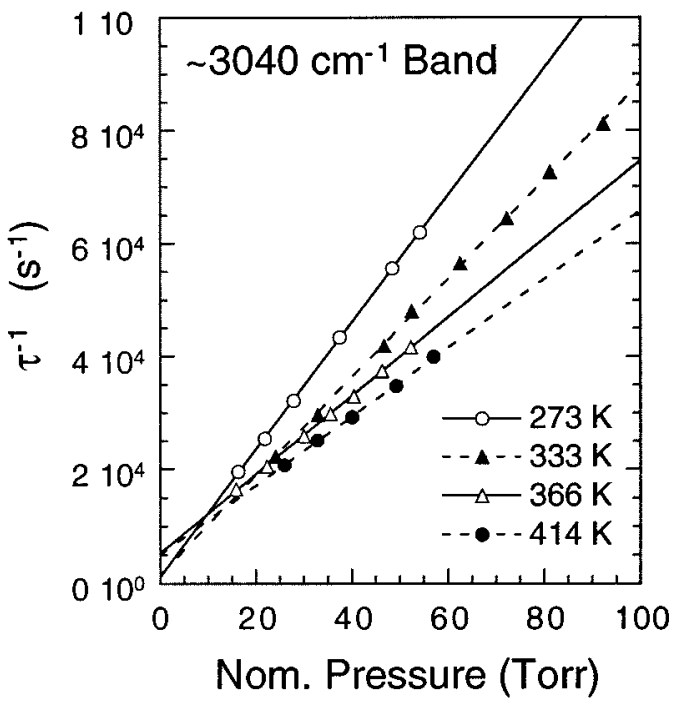

FIG. 3. Inverse time constants for IRF decay.

Typically, 5-15 experiments were performed at each temperature. Several methods were explored to minimize the effects of experimental fluctuations in pressure and other parameters. It was found that the effects of minor pressure fluctuations could be minimized by transforming the data to a set of reduced variables and then combining all the data for the final analysis. The reduced intensities were obtained by carrying out a nonlinear least squares analysis and normalizing as described above. The reduced time scale $t^{\prime}$ was defined by $t^{\prime}=t / \tau$, where $t$ is the recorded time and $\tau$ is the time at which the IRF intensity of the fundamental band drops to $I_{0} / e$ (according to the least squares fit). For a set of runs at a fixed temperature, a plot of $\tau^{-1}$ vs $N_{p}$ produces a straight line with slope $k_{b}$, as illustrated in Fig. 3 (note that the decay rates depend on bath gas temperature). The nonzero intercepts are due to small zero shifts in the electronic pressure transducer and only the slopes of the curves are significant. The reduced time scale is converted to a collision scale by noting that $Z=k_{\mathrm{LJ}} / k_{b}$. The transformation of variables forces all of the data sets to be expressed on the same basis. The transformed data for all the runs at a given temperature were combined and weighted according to the standard deviations of the individual runs. The combined data set for each temperature was fitted by nonlinear least squares and the results are presented in Table I. The statistical uncertainty associated with each datum is of the order of $\pm 1 \%$.

As described elsewhere, ${ }^{12,15,17,18}$ the bulk average energy $\langle\langle E(t)\rangle\rangle$ (averaged over the population distribution, which evolves with time) can be extracted approximately by using the time dependent observed intensity. To do this, the experimental intensity is identified with $I_{\mathrm{ci}}$ (the calculated intensity for band $i$ ) and the bulk average energy $\langle\langle E\rangle\rangle$ is identified with $E$ in the following equations for pyrazine:

$$
\begin{aligned}
& E_{1}=950+15010 I_{1}+8650 I_{1}^{0.5}+8180 I_{1}^{0.25}, \\
& E_{2}=4480+1320 I_{2}+8560 I_{2}^{0.5}+18390 I_{2}^{0.25},
\end{aligned}
$$

\begin{tabular}{|c|c|c|c|c|c|}
\hline \multirow[b]{2}{*}{$T(\mathrm{~K})$} & \multicolumn{2}{|c|}{$\sim 3040 \mathrm{~cm}^{-1}$ band } & \multicolumn{2}{|c|}{$\sim 6000 \mathrm{~cm}^{-1}$ band } & \multirow{2}{*}{$\begin{array}{l}10^{10} k_{\mathrm{LJ}} \\
\left(\mathrm{cm}^{3} \mathrm{~s}^{-1}\right)\end{array}$} \\
\hline & $100 k_{1}$ & $10^{4} b_{1}$ & $100 k_{2}$ & $10^{4} b_{2}$ & \\
\hline 254 & 5.96 & 4.06 & 7.97 & 12.20 & 6.49 \\
\hline 260 & 4.66 & 4.40 & $\ldots$ & $\ldots$ & 6.52 \\
\hline 273 & 4.21 & 3.09 & 6.62 & 6.47 & 6.57 \\
\hline 282 & 3.90 & 3.02 & $\ldots$ & $\ldots$ & 6.61 \\
\hline 294 & 3.94 & 2.46 & $\ldots$ & $\ldots$ & 6.65 \\
\hline 333 & 3.90 & 2.40 & 5.75 & 5.74 & 6.79 \\
\hline 366 & 3.45 & 1.51 & 5.66 & 2.31 & 6.90 \\
\hline 414 & 3.31 & 1.50 & 4.97 & 3.48 & 7.04 \\
\hline
\end{tabular}

TABLE I. IRF decay parameters. ${ }^{\mathrm{a}}$

${ }^{a}$ Normalized intensity: $I_{i}(Z)=\exp \left[-\left(k_{i} Z+b_{i} Z^{2}\right)\right]$, where $Z$ is the number of collisions and $i=1,2$ designates the fundamental and overtone bands, respectively. The statistical uncertainties in the table entries are $\sim 1 \%$.

where $I_{1}$ and $I_{2}$ refer to the fundamental $\mathrm{C}-\mathrm{H}$ stretch band near $3040 \mathrm{~cm}^{-1}$ and the overtone band near $6000 \mathrm{~cm}^{-1}$, respectively. Both intensities have been normalized to unity at $\sim 32760 \mathrm{~cm}^{-1}$ (the initial excitation energy, which includes thermal energy). These relationships are valid for the energy range from $\sim 5000$ to $\sim 35000 \mathrm{~cm}^{-1}$. The above relationships for pyrazine were calculated using a vibrational assignment ${ }^{29}$ and assuming that one rotational degree of freedom is active, ${ }^{30}$ as summarized elsewhere. ${ }^{26}$ To emphasize that this approximate technique has been used to invert the data, the subscript "inv" is appended: $\langle\langle E\rangle\rangle_{\text {inv }}$. The derivative of $\langle\langle E\rangle\rangle_{\text {inv }}$ with respect to $Z$ gives $\langle\langle\Delta E\rangle\rangle_{\text {inv }}$, the bulk average vibrational energy lost by pyrazine per collision. Plots of $\langle\langle\Delta E(E)\rangle\rangle_{\text {inv }}$ as a function of $\langle\langle E\rangle\rangle_{\text {inv }}$ are presented in Fig. 4 , where it is clear that the average energy transferred per collision depends on the bath gas temperature, as well as on vibrational energy. Further and more accurate analysis requires numerical master equation simulations of the experiments.

\section{Master equation simulations}

In the present work, energy transfer parameters were obtained by numerical master equation simulations of the ex-

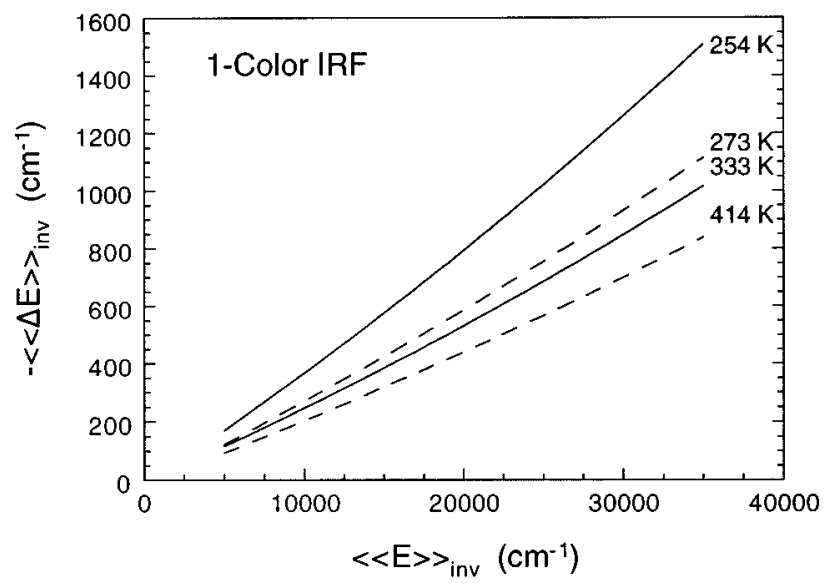

FIG. 4. Bulk average energy transfer step sizes derived from the fundamental band $\left(\sim 3040 \mathrm{~cm}^{-1}\right)$ emission. 
perimental IRF data. The stochastic master equation formulation used in the present work has been described in detail elsewhere. ${ }^{31,32}$ Basically, it employs an empirical step size model and Monte Carlo techniques to select the initial conditions and the progress of each stochastic trial, based on microscopic reversibility and detailed balance. As each simulation progresses, the IRF intensity of each emission band is calculated according to the theoretical expression relating the IRF intensity to bulk average energy, which has been described and tested elsewhere. ${ }^{7,12,16,17,27}$ The procedure was first to obtain an estimate of the energy transfer parameters directly from the experimental data and then to refine the parameters by comparing the calculated and experimental IRF intensities.

The experimental data and results presented in Figs. 2 and 4 show that the deactivation proceeds much more rapidly at low temperatures. In large part, this effect is due to the competition between activating and deactivating collisions. At low temperatures, activating collisions are much less important than at higher temperatures and the net rate of deactivation is increased. The rates of activation and deactivation are related through detailed balance and thus specification of the collision step size distribution $P\left(E^{\prime}, E, T\right)$ for deactivating collisions also specifies that for the activating collisions. This "thermodynamic" dependence on temperature can obscure more subtle effects and thus it is preferable to discuss $P\left(E^{\prime}, E, T\right)$ and model parameters, rather than the total rate of deactivation. ${ }^{9,33}$

The collision step size distribution function for large molecule deactivation is not known and simple empirical functions are chosen primarily for convenience. In the present work, we have used the exponential and biexponential models. The exponential model for down-steps is given by

$$
P\left(E^{\prime}, E, T\right)=M(E) \exp \left[-\left(E-E^{\prime}\right) / \alpha(E, T)\right], \quad E^{\prime}<E,
$$

where $\alpha(E)$ is an energy-dependent parameter which is almost identical to $\langle\Delta E\rangle_{d}$, the average energy transferred in deactivating collisions, and $M(E)$ is a normalization constant, which depends on energy. The parameter $\alpha(E, T)$ was assumed to have the functional form

$$
\alpha(E, T)=c_{0}+c_{1} E+c_{2} E^{2},
$$

where $c_{0}, c_{1}$, and $c_{2}$ are (temperature-dependent) empirical coefficients. As described elsewhere, ${ }^{26}$ initial estimates for the coefficients can be obtained by least squares fitting the data for $\langle\langle\Delta E\rangle\rangle_{\text {inv }}$ vs $\langle\langle E\rangle\rangle_{\text {inv }}$. By adjusting the coefficients, it is possible to achieve nearly exact agreement between simulation and experiment for a single IRF band. However, we found it impossible to fit two IRF bands simultaneously using a single exponential model. For the purpose of simulating the two-color results, a biexponential model was necessary:

$$
\begin{aligned}
P\left(E^{\prime}, E, T\right)= & M(E)\left\{\left(1-f_{c}(T)\right) \exp \left[-\left(E-E^{\prime}\right) / \alpha(E, T)\right]\right. \\
& \left.+f_{c}(T) \exp \left[-\left(E-E^{\prime}\right) / \beta\right]\right\}, \quad E^{\prime}<E
\end{aligned}
$$

\begin{tabular}{|c|c|c|c|c|c|c|c|}
\hline \multirow[b]{2}{*}{$T(\mathrm{~K})$} & \multicolumn{3}{|c|}{ one-color IRF } & \multicolumn{4}{|c|}{ two-color IRF } \\
\hline & $c_{0}$ & $100 c_{1}$ & $-10^{7} c_{2}$ & $c_{0}$ & $100 c_{1}$ & $-10^{7} c_{2}$ & $10^{3} f_{c}$ \\
\hline 254 & 75 & 4.00 & 1.0 & 75 & 4.0 & 7.0 & 4.0 \\
\hline 260 & 100 & 3.70 & 0.6 & $\ldots$ & $\ldots$ & $\ldots$ & $\cdots$ \\
\hline 273 & 90 & 3.60 & 3.0 & 75 & 3.9 & 8.0 & 2.3 \\
\hline 282 & 120 & 2.90 & 1.0 & $\ldots$ & $\ldots$ & $\ldots$ & $\ldots$ \\
\hline 294 & 100 & 3.05 & 1.0 & $\ldots$ & $\ldots$ & $\ldots$ & $\ldots$ \\
\hline 333 & 100 & 3.10 & 1.0 & 75 & 3.9 & 9.0 & 2.2 \\
\hline 366 & 125 & 2.70 & 1.0 & 50 & 3.0 & 4.5 & 1.8 \\
\hline 414 & 150 & 2.75 & 1.5 & 75 & 4.0 & 9.0 & 1.8 \\
\hline
\end{tabular}

TABLE II. Energy transfer parameters from master equation simulations (see text for details)

where $f_{c}(T)$ and $\beta$ are empirical parameters. The biexponential model was used in fitting the two-color IRF results and, as in earlier work, ${ }^{15}$ it was found empirically that a constant $\beta=20000 \mathrm{~cm}^{-1}$ gave satisfactory agreement with the experimental data; smaller values of $\beta$ did not produce satisfactory simulations and no energy dependence was required for satisfactory agreement. Strategies for carrying out the simulations are described in the earlier work. ${ }^{26}$

\section{Simulation results}

The fundamental band emissions at all experimental temperatures were measured with good signal/noise ratios, but some of the overtone band data were too noisy to be useful. Thus, one-color simulations were carried out for all experimental temperatures and two-color simulations were carried out when both fundamental and overtone emissions were available. The results of the simulations are presented in Table II. These energy transfer parameters, although not necessarily unique, provide the best current description of pyrazine deactivation by unexcited pyrazine. They are model dependent in the sense that exponential and biexponential models were used for the collision step-size distribution, but the energy transfer temperature dependence can be established within the framework of these models.

Inspection of the results in Table II shows that the individual parameters show relatively little systematic temperature dependence, although there are experimental fluctuations. In particular, the two-color results for $T=366 \mathrm{~K}$ show large excursions of $c_{2}$ and $f_{c}$ from the mean values, but the two parameters are highly correlated and the resulting values for $\alpha(E, T)$ are reasonably consistent with the results at other temperatures.

Since two different collision step size models are employed in the present work and the $\alpha(E, T)$ parameters are not directly comparable, it is instead useful to compare values for $\langle\Delta E\rangle_{d}$, the average energy transferred in deactivating collisions:

$$
\langle\Delta E(T)\rangle_{d}=\frac{\int_{0}^{E}\left(E-E^{\prime}\right) P\left(E^{\prime}, E, T\right) d E^{\prime}}{\int_{0}^{E} P\left(E^{\prime}, E, T\right) d E^{\prime}} .
$$

In Fig. 5, the results for $\langle\Delta E(T)\rangle_{d}$ determined from the onecolor and two-color analyses are presented for comparison (the lines in the figure are intended to guide the eye and have 


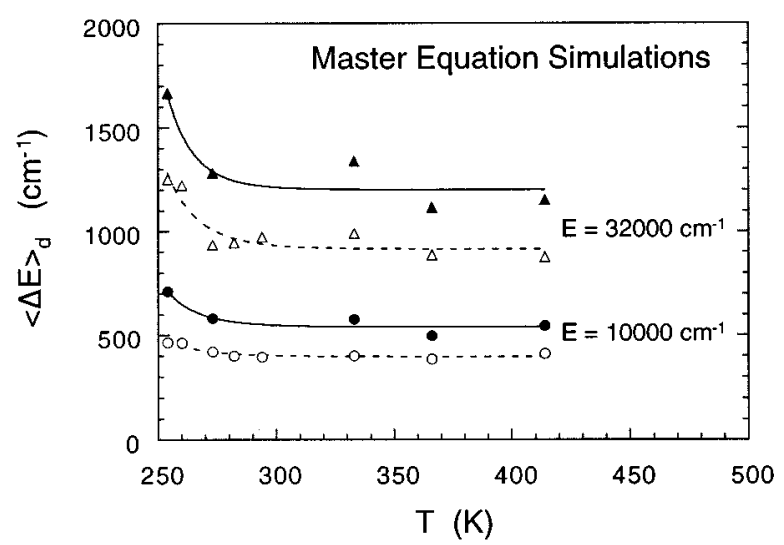

FIG. 5. Average energies transferred per deactivating collision $\left(\langle\Delta E\rangle_{d}\right)$ as functions of vibrational energy $\left(10000 \mathrm{~cm}^{-1}\right.$ and $\left.32000 \mathrm{~cm}^{-1}\right)$ and temperature. Solid lines and filled points: two-color IRF; broken lines and open points: one-color IRF.

no special significance). Although the one-color results are systematically displaced from the more accurate two-color values, both depend on temperature in nearly the same way.

From the simulations, it also possible to calculate the bulk average energy transferred per collision, $\langle\langle\Delta E\rangle\rangle$, as a function of both temperature and the bulk average energy, $\langle\langle E\rangle\rangle$. The results for three values of $\langle\langle E\rangle\rangle$ are presented as functions of temperature in Fig. 6 for the two-color IRF data. These results can be compared with those obtained using the simple inversion technique described above (see Fig. 4).

\section{Discussion}

\section{Comparison with other systems}

The results show that $\alpha(E, T)$ and $\langle\Delta E(T)\rangle_{d}$ for both models at each vibrational energy are nearly constant at high temperatures, but increase significantly below $\sim 300 \mathrm{~K}$. The constant values at higher temperatures are consistent with the earlier IRF and UVA experiments, ${ }^{5-7}$ but they contrast sharply with the recent experiments carried out with excited alkyl free radicals. ${ }^{1-4}$ The reasons for this difference are not

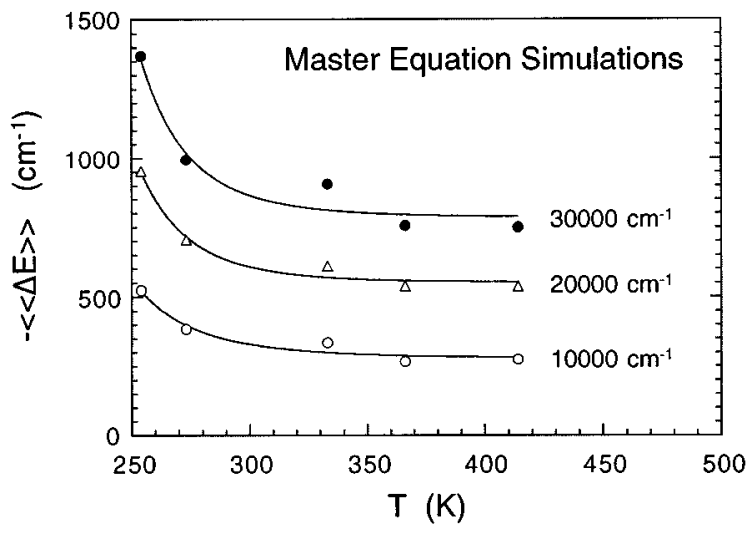

FIG. 6. Bulk average energy transferred per collision $(\langle\langle\Delta E\rangle\rangle)$ as functions of bulk average vibrational energy and temperature. These are simulations of the two-color IRF data. known, but there are at least two major differences between the present system and the alkyl radical systems. One difference is that the alkyl radical systems involved deactivation by small collider gases, rather than the polyatomic "parent." With small colliders, vibration-to-translation and rotation $(\mathrm{V}-\mathrm{T} / \mathrm{R})$ energy transfer is dominant. $^{18}$ In contrast, vibration-to-vibration $(\mathrm{V}-\mathrm{V})$ energy transfer may dominate in deactivation by the parent: classical trajectory calculations show that in the deactivation of excited benzene by unexcited benzene, which is similar to the present system, $\mathrm{V}-\mathrm{V}$ energy transfer dominates. ${ }^{34}$

Another possible reason why the alkyl radicals may behave differently from the pyrazine system is because of the qualitative differences between these classes of molecular species. The alkyl radicals have lower vibrational frequencies and more internal rotors than the unsaturated species. The electronic ground state of alkyl free radicals is doubly degenerate, while that of the unsaturated compounds is singly degenerate: it is known from other pyrazine studies that triplet state pyrazine undergoes more rapid vibrational deactivation than the singlet ground state. ${ }^{22,35}$ Studies with small molecules also show qualitative differences in energy transfer when excited electronic states are involved. ${ }^{36,37}$ If the same differences are operative with the doubly degenerate alkyl radical ground electronic state, then they may explain the different temperature dependences.

Unfortunately, the UVA and IRF experiments require optical pumping and fast radiationless transitions to prepare the vibrationally excited species, and these requirements are met by the unsaturated compounds, but not by alkanes and alkyl free radicals. Thus, application of the same experimental technique to both types of species will be very difficult or impossible. This is a very important problem, however, and future work should address this issue.

\section{Details of the temperature dependence}

At higher temperatures, $\alpha(E, T)$ and $\langle\Delta E(T)\rangle_{d}$ are both nearly constant, but both are larger at lower temperatures (see Fig. 5). Similar behavior is observed for $\langle\langle\Delta E\rangle\rangle$, which includes both up and down transitions (see Fig. 6). $\langle\langle\Delta E\rangle\rangle$ becomes smaller at higher temperatures, because the down transitions are more effectively counter balanced by the up transitions. Taking all the data points in Figure 6 into account, $\langle\langle\Delta E\rangle\rangle$ is proportional to $T^{-1}$, but if the $254 \mathrm{~K}$ datum is neglected, the proportionality is $T^{-0.7}$. These results can be compared with trajectory calculations on the deactivation of excited toluene by helium, where $\langle\langle\Delta E\rangle\rangle \sim T^{0.44}{ }^{38}$ The fact that the present results have an opposite dependence on temperature may indicate that $\mathrm{V}-\mathrm{T} / \mathrm{R}$ transfer is not the dominant deactivation mechanism for pyrazine* + pyrazine collisions.

The temperature dependence of $\alpha(E, T)$ and $\langle\Delta E(T)\rangle_{d}$ is suggestive of a change in mechanism at lower temperatures. The estimated Lennard-Jones well depth of pyrazine is $\sim 436 \mathrm{~K}$ (Ref. 22) and the increase in $\alpha(E, T)$ and $\langle\Delta E(T)\rangle_{d}$ occurs below about $275 \mathrm{~K}$, where many collisions may produce longer-lived complexes. It seems plausible that 


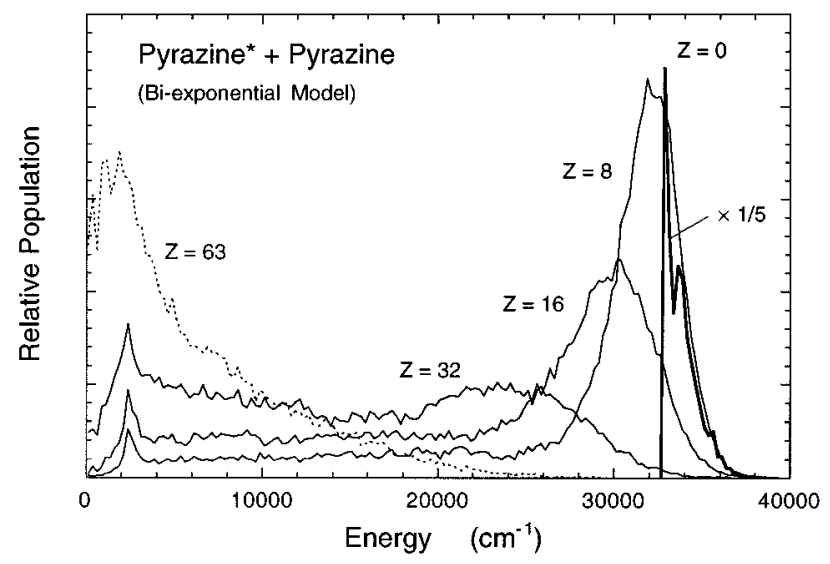

FIG. 7. Simulated population distribution as a function of the number of collisions $(Z)$ at $273 \mathrm{~K}$. The fluctuations are due to noise in the stochastic master equation results.

$\mathrm{V}-\mathrm{V}$ transfer will become more efficient at lower temperatures, when longer lived complexes and chattering collisions $^{39-41}$ are more prevalent. In contrast, V-TR energy transfer is more effective in higher velocity impulsive collisions and thus should be less important at the lower temperatures. $^{38}$

\section{Evolution of the population distribution and "super collisions"'}

During the collisional cascade, the energy distribution of excited molecules evolves with time, as illustrated by the master equation simulation results presented in Fig. 7. The simulation was carried out for $414 \mathrm{~K}$ with the two-color energy transfer parameters in Table II. Before any collisions occur $(Z=0)$, the thermal Boltzmann distribution is promoted by absorption of a photon. The structure apparent at $Z=0$ in Fig. 7 is due to the thermal population distribution among the low-lying vibrational states of pyrazine. As the pyrazine experiences collisions, the distribution spreads and cascades down the energy ladder, eventually collapsing back to a Boltzmann distribution corresponding to $\sim 455 \mathrm{~K}$, since the absorbed laser energy produces a temperature increase. The calculated temperature increase was included in the master equation simulations by assuming that $1 \%$ of the pyrazine was excited by the laser, but even if it had been neglected, the effect would be negligible, because the energy transfer parameters are only weakly dependent on temperature.

The second component of the bi-exponential model gives the contribution due to "super collisions,"' which are defined in various ways by different authors. ${ }^{42-45}$ Perhaps the most precise definition is that "super collisions" are energy transfer steps which occur with probabilities greater than predicted by a simple exponential model. Since the exponential model is merely a mathematical convenience and has no compelling theoretical basis, "super collisions" do not necessarily describe a special phenomenon, although classical trajectory calculations have found that certain special types of collisions contribute. ${ }^{44-47}$ The empirical biexponential model is chosen for convenience, rather than on the basis of

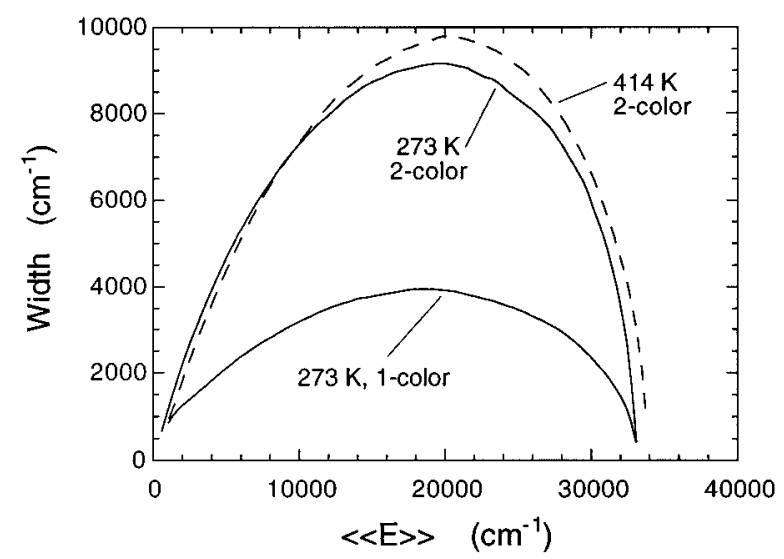

FIG. 8. Widths of the simulated population distributions.

theory, and other models may be just as useful for master equation simulations. More experimental work is needed to better define the collision step size distribution and more theoretical work is needed to better define its preferred functional form.

Pragmatically, the effect of the second component of the biexponential model ("super collisions") is to skew and distort the cascading population distribution, even though $f_{c}(T)$ is small. The cumulative effect of successive collisions is to create a low energy "tail" on the population distribution, as shown in Fig. 7. This "tail" greatly increases the width of the population distribution [defined by $\sigma(t)$, the square root of the variance of the distribution function at time $t]$, as shown in Fig. 8. Higher moments of the population distribution are also strongly affected by "super collisions," but two-color IRF measurements provide most information on only the first two moments of the distribution, and so higher order effects are not as well determined. The effect of the second component of the biexponential model is clearly apparent in Fig. 8, where the one-color IRF simulation is compared with the two-color simulation. Both simulations fit the data for the fundamental IRF band, but only the biexponential model fits both bands. The second exponential component essentially doubles the width of the distribution function, and it will affect incubation times and reaction yields in both steady-state and nonequilibrium systems. ${ }^{20,45}$

Super collisions appear to be ubiquitous, since they have been observed in almost every system capable of detecting them. The multicolor IRF technique is no exception and a biexponential model is needed to describe multicolor IRF results for $\mathrm{C}_{6} \mathrm{H}_{6}$ and $\mathrm{C}_{6} \mathrm{D}_{6},{ }^{15,20} 1,3,5-\mathrm{C}_{6} \mathrm{H}_{3} \mathrm{D}_{3}, \mathrm{CH}_{3} \mathrm{C}_{6} \mathrm{D}_{5}$, and $\mathrm{CD}_{3} \mathrm{C}_{6} \mathrm{H}_{5},{ }^{19}$ as well as pyrazine.

\section{CONCLUSIONS}

The experimental data and analysis presented in this paper show that energy transfer in the deactivation of excited pyrazine by unexcited pyrazine is only weakly dependent on temperature. At low temperatures, the increase in $\langle\Delta E\rangle_{d}$ may indicate the effects of the Lennard-Jones attractive well and a change in energy transfer mechanism. At high tem- 
peratures, $\langle\Delta E\rangle_{d}$ is essentially constant, perhaps indicating that $\mathrm{V}-\mathrm{T} / \mathrm{R}$ energy transfer is not dominant, since it is expected to be enhanced at higher temperatures. It seems likely that $\mathrm{V}-\mathrm{V}$ transfer is the dominant mechanism, as indicated in classical trajectory calculations on benzene, ${ }^{34}$ which is very similar in behavior to pyrazine. $^{26}$

\section{ACKNOWLEDGMENTS}

Thanks go to the Office of Basic Energy Sciences at the U.S. Department of Energy for funding. L.A.M. thanks the Regents of the University of Michigan for a Regents Fellowship and C.D.C. thanks the College of Engineering for a Sarah Marian Parker scholarship.

${ }^{1}$ Y. Feng, J. T. Niiranen, A. Bencsura, V. D. Knyazev, and D. Gutman, J. Phys. Chem. 97, 871 (1993).

${ }^{2}$ M. A. Hanning-Lee, N. J. B. Green, M. J. Pilling, and S. H. Robertson, J. Phys. Chem. 97, 860 (1993).

${ }^{3}$ V. D. Knyazev, I. A. Dubinsky, I. R. Slagle, and D. Gutman, J. Phys. Chem. 98, 5279 (1994).

${ }^{4}$ V. D. Knyazev, I. A. Dubinsky, I. R. Slagle, and D. Gutman, J. Phys. Chem. 98, 11099 (1994).

${ }^{5}$ H. Hippler, J. Troe, and H. J. Wendelken, J. Chem. Phys. 78, 6709 (1983).

${ }^{6}$ M. Heymann, H. Hippler, and J. Troe, J. Chem. Phys. 80, 1853 (1984).

${ }^{7}$ J. R. Barker and R. E. Golden, J. Phys. Chem. 88, 1012 (1984).

${ }^{8}$ V. D. Knyazev, J. Phys. Chem. 99, 14738 (1995).

${ }^{9}$ D. C. Tardy and B. S. Rabinovitch, Chem. Rev. 77, 369 (1977).

${ }^{10} \mathrm{H}$. Hippler and J. Troe, in Bimolecular Collisions, edited by M. N. R. Ashford and J. E. Bagott (Royal Society of Chemistry, London, 1989), p. 209.

${ }^{11}$ I. Oref and D. C. Tardy, Chem. Rev. 90, 1407 (1990).

${ }^{12}$ J. R. Barker and B. M. Toselli, Int. Rev. Phys. Chem. 12, 305 (1990).

${ }^{13}$ M. J. Rossi, J. R. Pladziewicz, and J. R. Barker, J. Chem. Phys. 78, 6695 (1983).

${ }^{14}$ J. Shi and J. R. Barker, J. Chem. Phys. 88, 6219 (1988).

${ }^{15}$ J. D. Brenner, J. P. Erinjeri, and J. R. Barker, Chem. Phys. 175, 99 (1993).

${ }^{16}$ B. M. Toselli and J. R. Barker, J. Chem. Phys. 97, 1809 (1992).

${ }^{17}$ B. M. Toselli, J. D. Brenner, M. L. Yerram, W. E. Chin, K. D. King, and J. R. Barker, J. Chem. Phys. 95, 176 (1991).
${ }^{18}$ B. M. Toselli and J. R. Barker, J. Chem. Phys. 95, 8108 (1991).

${ }^{19}$ J. R. Barker, J. Monat, L. A. Miller, J. D. Brenner, V. Buller, K. Pohl, and K. D. King (unpublished).

${ }^{20}$ J. R. Barker, J. D. Brenner, and B. M. Toselli, Adv. Chem. Kin. Dyn. 2B, 393 (1995).

${ }^{21}$ J. Knee and P. Johnson, J. Phys. Chem. 89, 948 (1985).

${ }^{22}$ T. J. Bevilacqua and R. B. Weisman, J. Chem. Phys. 98, 6316 (1993).

${ }^{23}$ O. Sneh, D. Dünn-Kittenplon, and O. J. Cheshnovsky, Chem. Phys. 91, 7331 (1989).

${ }^{24}$ J. D. Chesko, D. Stranges, A. G. Suits, and Y. T. Lee, J. Chem. Phys. 103, 6290 (1995).

${ }^{25}$ J. Kommandeur, W. A. Majewski, W. L. Meerts, and D. W. Pratt, Ann. Rev. Phys. Chem. 38, 433 (1987).

${ }^{26}$ L. A. Miller and J. R. Barker, J. Chem. Phys. (to be published).

${ }^{27}$ J. F. Durana and J. D. McDonald, J. Chem. Phys. 64, 2518 (1977).

${ }^{28}$ C. A. Michaels, H. C. Tapalian, Z. Lin, E. T. Sevy, and G. W. Flynn, Faraday Disc. (to be published).

${ }^{29}$ K. B. Hewett, M. Shen, C. L. Brummel, and L. A. Philips, J. Chem. Phys. 100, 4077 (1994).

${ }^{30}$ K. K. Innes, J. P. Byrne, and I. G. Ross, J. Mol. Spectrosc. 22, 125 (1967).

${ }^{31}$ J. R. Barker, Chem. Phys. 77, 301 (1983).

${ }^{32}$ J. R. Barker and K. D. King, J. Chem. Phys. 103, 4953 (1995), and references therein.

${ }^{33}$ R. G. Gilbert, Chem. Phys. Lett. 96, 259 (1983).

${ }^{34}$ T. Lenzer and K. Luther, J. Chem. Phys. 104, 3391 (1996).

${ }^{35}$ R. B. Weisman, Adv. Chem. Kin. Dyn. 2B, 333 (1995).

${ }^{36}$ B. M. Toselli, T. L. Walunas, and J. R. Barker, J. Chem. Phys. 92, 4793 (1990)

${ }^{37}$ G. V. Hartland, D. Qin, and H.-L. Dai, J. Chem. Phys. 102, 8677 (1995).

${ }^{38}$ K. F. Lim, J. Chem. Phys. 100, 7385 (1994); 101, 8756 (1994).

${ }^{39}$ K. F. Lim and R. G. Gilbert, J. Phys. Chem. 94, 72 (1990); 94, 77 (1990).

${ }^{40}$ R. G. Gilbert, Int. Rev. Phys. Chem. 10, 319 (1991).

${ }^{41}$ V. Bernshtein and I. Oref, J. Chem. Phys. 104, 1958 (1996).

${ }^{42}$ S. Hassoon, I. Oref, and C. Steel, J. Chem. Phys. 89, 1743 (1988).

${ }^{43}$ I. M. Margulis, S. S. Sapers, C. Steel, and I. Oref, J. Chem. Phys. 90, 923 (1989).

${ }^{44}$ D. L. Clarke, K. G. Thompson, and R. G. Gilbert, Chem. Phys. Lett. 182, 357 (1991).

${ }^{45}$ I. Oref, Adv. Chem. Kin. Dyn. 2B, 285 (1995).

${ }^{46}$ G. Lendvay and G. C. Schatz, J. Phys. Chem. 94, 8864 (1990).

${ }^{47}$ G. Lendvay and G. C. Schatz, Adv. Chem. Kin. Dyn. 2B, 481 (1995). 